\title{
A CHAINABLE CONTINUUM NOT HOMEOMORPHIC TO AN INVERSE LIMIT ON $[0,1]$ WITH ONLY ONE BONDING MAP
}

\author{
WILLIAM S. MAHAVIER
}

Introduction. In this note by a continuum we mean a nondegenerate, compact, connected metric space. It is known (see [4] or [6]) that each chainable continuum is homeomorphic to the inverse limit of a sequence of maps from $[0,1]$ on to $[0,1]$, and it is not difficult to show, conversely, that if each of $f_{1}, f_{2}, \cdots$ maps $[0,1]$ on $[0,1]$, then the inverse limit of this sequence is a chainable continuum. G. W. Henderson has recently shown [5] that there is a map of $[0,1]$ on $[0,1]$ such that the inverse limit with it as the only bonding map is a pseudo arc. We observe that not every chainable continuum can be so represented (using only one bonding map) but that each chainable continuum can be embedded in such an inverse limit.

If each term of the sequence $\alpha=\left\{f_{1}, f_{2}, \cdots\right\}$ maps $[0,1]$ on $[0,1]$ then the inverse limit of the sequence $\alpha$, denoted by $\lim \alpha$, is the subspace of the infinite cartesian product $[0,1]^{\infty}$ consisting of all number sequences $x_{1}, x_{2}, \cdots$ such that for each positive in teger $i$, $f_{i}\left(x_{i+1}\right)=x_{i}$. If $f$ maps $[0,1]$ on $[0,1]$, then $\lim f$ denotes $\lim \alpha$ where $\alpha=\{f, f, \cdots\}$. For a discussion of properties of inverse limit spaces see $[3]$.

General results. We first observe

TheOREM 1. If $f$ maps $[0,1]$ on $[0,1]$, then there is a nontrivial homeomorphism of $\lim f$ on $\lim f$.

For if $f$ is such a map, then for each point $x_{1}, x_{2}, \ldots$ in $\lim f$ we define $\phi\left(x_{1}, x_{2}, \cdots\right)=f\left(x_{1}\right), f\left(x_{2}\right), \cdots=f\left(x_{1}\right), x_{1}, x_{2}, \cdots$ and it follows by direct argument that $\phi$ is a homeomorphism of $\lim f$ on $\lim f$. Moreover, $\phi$ is nontrivial unless $f$ is the identity map in which case $\lim f$ is an arc and clearly there is then a nontrivial homeomorphism of $\lim f$ onto itself. As an immediate consequence of Theorem 1 we have

ExAmple 1. A chainable continuum $M$ such that if $f$ maps $[0,1]$ on $[0,1]$, then $M$ is not homeomorphic to $\lim f$.

Let $M$ denote a chainable continuum such that no two of its subcontinua are homeomorphic (see [1]). That $M$ is not homeomorphic to $\lim f$ for any map $f$ of $[0,1]$ on $[0,1]$ follows from the fact that

Received by the editors December 15, 1964. 
if $\phi$ is a nontrivial homeomorphism of $M$ onto $M$, then $M$ contains a subcontinuum $H$ such that $\phi(H)$ is different from $H$.

In order that a chainable continuum be representable as an inverse limit with only one bonding map it is not sufficient that it support a nontrivial homeomorphism as may be seen by

EXAMPLE 2. A chainable continuum $M$ such that (1) if $f$ maps $[0,1]$ on $[0,1]$, then $\lim f$ is not homeomorphic to $M$ and (2) there is a nontrivial homeomorphism of $M$ on $M$.

-R. Bennett has shown [2] that if $A^{\prime}$ is a chainable continuum, there is a chainable continuum $B$ which is the union of a topological ray $R$ and a continuum $A$ homeomorphic to $A^{\prime}$ such that $A \cap R=0$ and $R$ is dense in $A$. Thus there is a chainable continuum $M$ which is the union of a topological ray $R$ and a chainable continuum $H$ such that (1) no two subcontinua of $H$ are homeomorphic, (2) $R$ does not intersect $H$, and (3) $R$ is dense in $H$. Clearly there are nontrivial homeomorphisms of $M$ on $M . M$ is not $\lim f$ for any map $f$ of $[0,1]$ on $[0,1]$ since if it were, then a homeomorphism $\phi$ as described in the proof of Theorem 1 could be defined. $\phi$ would map $H$ in to $M$ but, since $\phi(H)$ contains no arc, $\phi(H) \cap R=0$, and thus $\phi(H)=H$. But this would imply that $\phi$ is the identity on $H$, that $f$ is the identity on some subinterval of $\mathrm{I}$ and that $H$ contains an arc. It follows that $M$ is not homeomorphic to $\lim f$ for any map $f$ of $[0,1]$ on $[0,1]$.

Finally we note that

THEOREM 2. If $M$ is a chainable continuum, there is a map $f$ of $[0,1]$ on $[0,1]$ such that $\lim f$ contains a continuum homeomorphic to $M$.

Let $M$ denote a chainable continuum and $\alpha=\left\{f_{1}, f_{2}, \cdots\right\}$ a sequence of maps of $[0,1]$ on $[0,1]$ such that $M$ is homeomorphic to $\lim \alpha$. We shall describe an $f$ such that $\lim f$ contains a homeomorphic image of $M$ by considering a collection of "copies" of the maps $f_{1}, f_{2}, \ldots$ in the disc $[0,1] \times[0,1]$ placed in such a way that the domain of the "copy" of $f_{i}$ is the range of the "copy" of $f_{i+1}$ for $i=1,2, \cdots$. Suppose $0<x_{1}<x_{2}<1,0<y_{1}<y_{2}<1$, and $i$ is a positive in teger. By the copy of $f_{i}$ in $\left[x_{1}, x_{2}\right] \times\left[y_{1}, y_{2}\right]$ we mean the map $h$ of $\left[x_{1}, x_{2}\right]$ on $\left[y_{1}, y_{2}\right]$ defined by

$$
h(x)=y_{1}+\left(y_{2}-y_{1}\right) f_{i}\left[\left(x-x_{1}\right) /\left(x_{2}-x_{1}\right)\right] \text { for } x \text { in }\left[x_{1}, x_{2}\right] .
$$

For each nonnegative integer $i$, let $a_{i}=1 / 2^{i+1}$ and $b_{i}=3 / 2^{i+2}$; and for each positive integer $i$, let $h_{i}$ denote the copy of $f_{i}$ in $\left[a_{i}, b_{i}\right]$ $\times\left[a_{i-1}, b_{i-1}\right]$. Let $f$ denote the map from $[0,1]$ on $[0,1]$ such that (1) $f(0)=0$ and $f(1)=1$, (2) if $x$ is in $\left[a_{i}, b_{i}\right]$ for some positive in teger $i$, $f(x)=h_{i}(x)$, and $(3) f$ is linear on each component of $[0,1] \backslash \bigcup_{i=1}^{\infty}\left[a_{i}, b_{i}\right]$. 
The subset of $\lim f$ consisting of those points $x_{0}, x_{1}, \cdots$ of $\lim f$, such that for each nonnegative in teger $i, x_{i}$ is in $\left[a_{i}, b_{i}\right]$, is homeomorphic to $M$.

\section{REFERENCES}

1. J. J. Andrews, A chainable continuum no two of whose nondegenerate subcontinua are homeomorphic, Proc. Amer. Math. Soc. 12 (1961), 333-334.

2. R. Bennett, On inverse limit sequences, M.A. thesis, University of Tennessee, Knoxville, Tenn., 1962, p. 45.

3. S. Eilenberg and N. Steenrod, Foundations of algebraic topology, Chapter VIII, Princeton Univ. Press, Princeton, N. J., 1952.

4. H. Freudenthal, Entwicklungen von Räumen und ihren Gruppen, Compositio Math. 4 (1937), 145-234.

5. G. W. Henderson, The pseudo-arc as an inverse limit with one binding map, Duke Math J. 31 (1964), 421-425.

6. Sibe Mardešić, On covering dimension and inverse limits of compact spaces, Illinois J. Math. 4 (1960), 278-291.

EMORY UNIVERSITY 